\title{
Comprehensive molecular characterization of gastric adenocarcinoma
}

The Cancer Genome Atlas Research Network*

Gastric cancer is a leading cause of cancer deaths, but analysis of its molecular and clinical characteristics has been complicated by histological and aetiological heterogeneity. Here we describe a comprehensive molecular evaluation of 295 primary gastric adenocarcinomas as part of The Cancer Genome Atlas (TCGA) project. We propose a molecular classification dividing gastric cancer into four subtypes: tumours positive for Epstein-Barr virus, which display recurrent PIK3CA mutations, extreme DNA hypermethylation, and amplification of JAK2, CD274 (also known as PD-L1) and PDCD1LG2 (also known as PD-L2); microsatellite unstable tumours, which show elevated mutation rates, including mutations of genes encoding targetable oncogenic signalling proteins; genomically stable tumours, which are enriched for the diffuse histological variant and mutations of RHOA or fusions involving RHO-family GTPase-activating proteins; and tumours with chromosomal instability, which show marked aneuploidy and focal amplification of receptor tyrosine kinases. Identification of these subtypes provides a roadmap for patient stratification and trials of targeted therapies.

Gastric cancer was the world's third leading cause of cancer mortality in 2012, responsible for 723,000 deaths ${ }^{1}$. The vast majority of gastric cancers are adenocarcinomas, which can be further subdivided into intestinal and diffuse types according to the Lauren classification ${ }^{2}$. An alternative system, proposed by the World Health Organization, divides gastric cancer into papillary, tubular, mucinous (colloid) and poorly cohesive carcinomas ${ }^{3}$. These classification systems have little clinical utility, making the development of robust classifiers that can guide patient therapy an urgent priority.

The majority of gastric cancers are associated with infectious agents, including the bacterium Helicobacter pylori ${ }^{4}$ and Epstein-Barr virus (EBV). The distribution of histological subtypes of gastric cancer and the frequencies of $H$. pylori and EBV associated gastric cancer vary across the globe ${ }^{5}$. A small minority of gastric cancer cases are associated with germline mutation in E-cadherin $(\mathrm{CDH} 1)^{6}$ or mismatch repair genes ${ }^{7}$ (Lynch syndrome), whereas sporadic mismatch repair-deficient gastric cancers have epigenetic silencing of $M L H 1$ in the context of a $\mathrm{CpG}$ island methylator phenotype (CIMP) $)^{8}$. Molecular profiling of gastric cancer has been performed using gene expression or DNA sequencing ${ }^{9-12}$, but has not led to a clear biologic classification scheme. The goals of this study by The Cancer Genome Atlas (TCGA) were to develop a robust molecular classification of gastric cancer and to identify dysregulated pathways and candidate drivers of distinct classes of gastric cancer.

\section{Sample set and molecular classification}

We obtained gastric adenocarcinoma primary tumour tissue (fresh frozen) from 295 patients not treated with prior chemotherapy or radiotherapy (Supplementary Methods S1). All patients provided informed consent, and local Institutional Review Boards approved tissue collection. We used germline DNA from blood or non-malignant gastric mucosa as a reference for detecting somatic alterations. Non-malignant gastric samples were also collected for DNA methylation $(n=27)$ and expression $(n=29)$ analyses. We characterized samples using six molecular platforms (Supplementary Methods S2-S7): array-based somatic copy number analysis, whole-exome sequencing, array-based DNA methylation profiling, messenger RNA sequencing, microRNA (miRNA) sequencing and reverse-phase protein array (RPPA), with $77 \%$ of the tumours tested by all six platforms. Microsatellite instability (MSI) testing was performed on all tumour DNA, and low-pass ( $\sim 6 \times$ coverage $)$ whole genome sequencing on 107 tumour/germline pairs.

To define molecular subgroups of gastric cancer we first performed unsupervised clustering on data from each molecular platform (Supplementary Methods S2-S7) and integrated these results, yielding four groups (Supplementary Methods S10.2). The first group of tumours was significantly enriched for high EBV burden $\left(P=1.5 \times 10^{-18}\right)$ and showed extensive DNA promoter hypermethylation. A second group was enriched for MSI $\left(P=2.1 \times 10^{-32}\right)$ and showed elevated mutation rates and hypermethylation (including hypermethylation at the $M L H 1$ promoter). The remaining two groups were distinguished by the presence or absence of extensive somatic copy-number aberrations (SCNAs). As an alternative means to define distinct gastric cancer subgroups, we performed integrative clustering of multiple data types using iCluster ${ }^{13}$ (Supplementary Methods S10.3). This analysis again indicated that EBV, MSI and the level of SCNAs characterize distinct subgroups (Supplementary Fig. 10.3). Based upon these results from analysis of all molecular platforms, we created a decision tree to categorize the 295 gastric cancer samples into four subtypes (Fig. 1a, b) using an approach that could more readily be applied to gastric cancer tumours in clinical care. Tumours were first categorized by EBV-positivity (9\%), then by MSI-high status, hereafter called MSI (22\%), and the remaining tumours were distinguished by degree of aneuploidy into those termed genomically stable (20\%) or those exhibiting chromosomal instability (CIN; 50\%).

Evaluation of the clinical and histological characteristics of these molecular subtypes revealed enrichment of the diffuse histological subtype in the genomically stable group $\left(40 / 55=73 \%, P=7.5 \times 10^{-17}\right)$ (Fig. 1c), an association not attributable to reduced SCNA detection in low purity tumours (Supplementary Fig. 2.8). Each subtype was found throughout the stomach, but CIN tumours showed elevated frequency in the gastroesophageal junction/cardia $(65 \%, P=0.012)$, whereas most EBV-positive tumours were present in the gastric fundus or body $(62 \%$, $P=0.03)$. Genomically stable tumours were diagnosed at an earlier age (median age 59 years, $P=4 \times 10^{-7}$ ), whereas MSI tumours were diagnosed at relatively older ages (median 72 years, $P=5 \times 10^{-5}$ ). MSI patients tended to be female $(56 \%, P=0.001)$, but most EBV-positive

*A list of authors and affiliations appears at the end of the paper. 
a

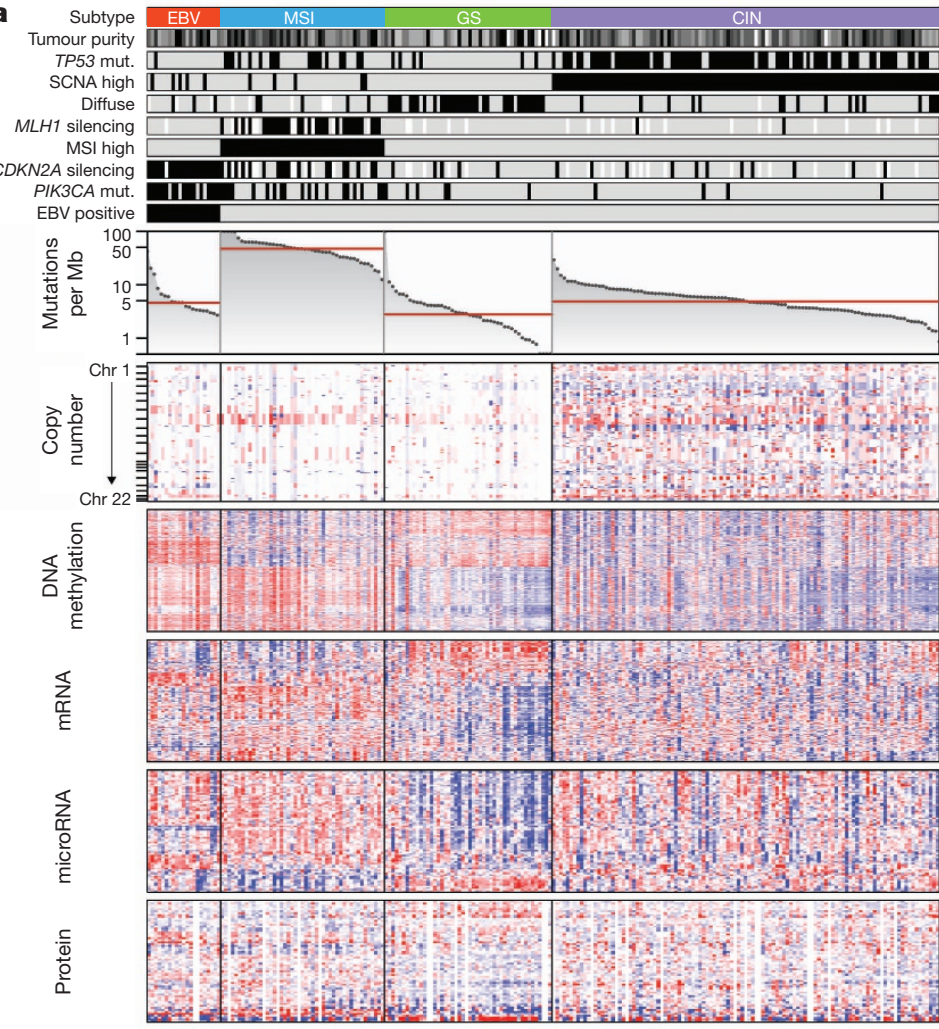

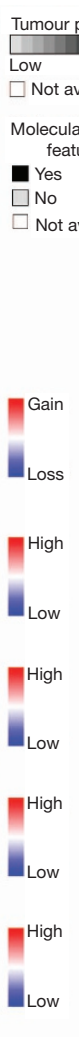

b

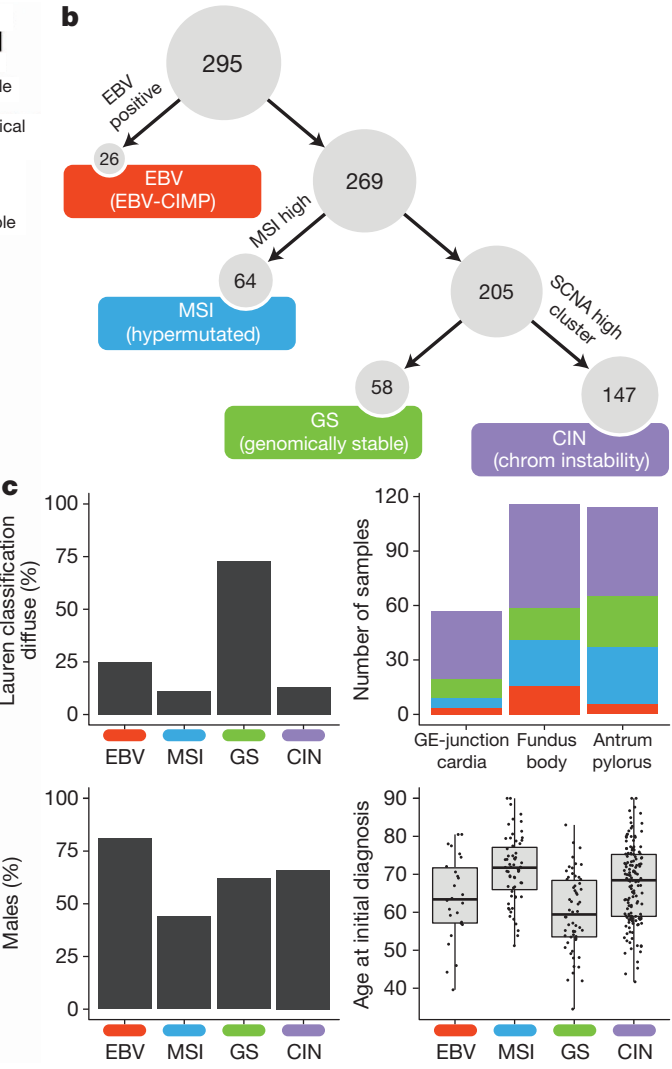

Figure $1 \mid$ Molecular subtypes of gastric cancer. a, Gastric cancer cases are divided into subtypes: Epstein-Barr virus (EBV)-positive (red), microsatellite instability (MSI, blue), genomically stable (GS, green) and chromosomal instability (CIN, light purple) and ordered by mutation rate. Clinical (top) and molecular data (top and bottom) from 227 tumours profiled with all six platforms are depicted. $\mathbf{b}$, A flowchart outlines how tumours were classified

cases were male $(81 \%, P=0.037)$, as previously reported ${ }^{14}$. We did not observe any systematic differences in distribution of subtypes between patients of East Asian and Western origin (Supplementary Methods S1.8). Initial outcome data from this cohort did not reveal survival differences between the four subgroups (Supplementary Information S1.7).

\section{EBV-associated DNA hypermethylation}

EBV is found within malignant epithelial cells in $9 \%$ of gastric cancers ${ }^{14}$. EBV status was determined using mRNA, miRNA, exome and wholegenome sequencing, yielding highly concordant results (Supplementary Fig. 9.7). By contrast, we detected only sporadic evidence of $H$. pylori, which may reflect the decline of bacterial counts accompanying the progression from chronic gastritis to subsequent carcinoma, as well as technical loss of luminal bacteria during specimen processing. Unsupervised clustering of $\mathrm{CpG}$ methylation performed on unpaired tumour samples revealed that all EBV-positive tumours clustered together and exhibited extreme CIMP, distinct from that in the MSI subtype ${ }^{8}$, consistent with prior reports ${ }^{15}$ (Fig. 2a). Differences between the EBV-CIMP and MSI-associated gastric-CIMP methylation profiles of tumours mirrored differences between these groups in their spectra of mutations (Fig. 1a) and gene expression (Supplementary Fig. 10.6a). EBV-positive tumours had a higher prevalence of DNA hypermethylation than any cancers reported by TCGA (Supplementary Fig. 4.6). All EBV-positive tumours assayed displayed $C D K N 2 A\left(p 16^{N K 4 A}\right)$ promoter hypermethylation, but lacked the $M L H 1$ hypermethylation characteristic of MSI-associated $\mathrm{CIMP}^{16}$. Genes with promoter hypermethylation most differentially silenced in EBV-positive gastric cancer are shown in Supplementary Table 4.3.

We observed strong predilection for PIK3CA mutation in EBVpositive gastric cancer as suggested by prior reports ${ }^{17,18}$, with non-silent into molecular subtypes. c, Differences in clinical and histological characteristics among subtypes with subtypes coloured as in $\mathbf{a}, \mathbf{b}$. The plot of patient age at initial diagnosis shows the median, 25th and 75th percentile values (horizontal bar, bottom and top bounds of the box), and the highest and lowest values within 1.5 times the interquartile range (top and bottom whiskers, respectively). GE, gastroesophageal.

PIK3CA mutations found in $80 \%$ of this subgroup $\left(P=9 \times 10^{-12}\right)$, including $68 \%$ of cases with mutations at sites recurrent in this data set or in the COSMIC repository. In contrast, 3 to $42 \%$ of tumours in the other subtypes displayed PIK3CA mutations. PI(3)-kinase inhibition therefore warrants evaluation in EBV-positive gastric cancer. PIK3CA mutations were more dispersed in EBV-positive cancers, but localized in the kinase domain (exon 20) in EBV-negative cancers (Fig. 2b). The most highly transcribed EBV viral mRNAs and miRNAs fell within the BamH1A region of the viral genome (Supplementary Fig. 9.8) and showed similar expression patterns across tumours, as reported separately ${ }^{19}$.

\section{Somatic genomic alterations}

To identify recurrently mutated genes, we analysed the 215 tumours with mutation rates below 11.4 mutations per megabase $(\mathrm{Mb})$ (none of which were MSI-positive) separately from the 74 'hypermutated' tumours. Within the hypermutated tumours, we excluded from analysis 11 cases with a distinctly higher mutational burden above 67.7 mutations per $\mathrm{Mb}$ (including one tumour with an inactivating POLE mutation ${ }^{20,21}$ ) (Supplementary Information S3.2-3.3), because their large numbers of mutations unduly influence analysis. We used the MutSigCV ${ }^{22}$ tool to define recurrent mutations in the 63 remaining hypermutated tumours by first evaluating only base substitution mutations, identifying 10 significantly mutated genes, including TP53, KRAS, ARID1A, PIK3CA, $E R B B 3, P T E N$ and $H L A-B$ (Supplementary Table 3.5). We found ERBB3 mutations in 16 of 63 tumours, with 13 of these tumours having mutations at recurrent sites or sites reported in COSMIC. MutSigCV analysis including insertions/deletions expanded the list of statistically significant mutated genes to 37, including RNF43, B2M and NF1 (Supplementary Fig. 3.9). Similarly, HotNet analysis of genes mutated within MSI tumours revealed common alterations in major histocompatibility 

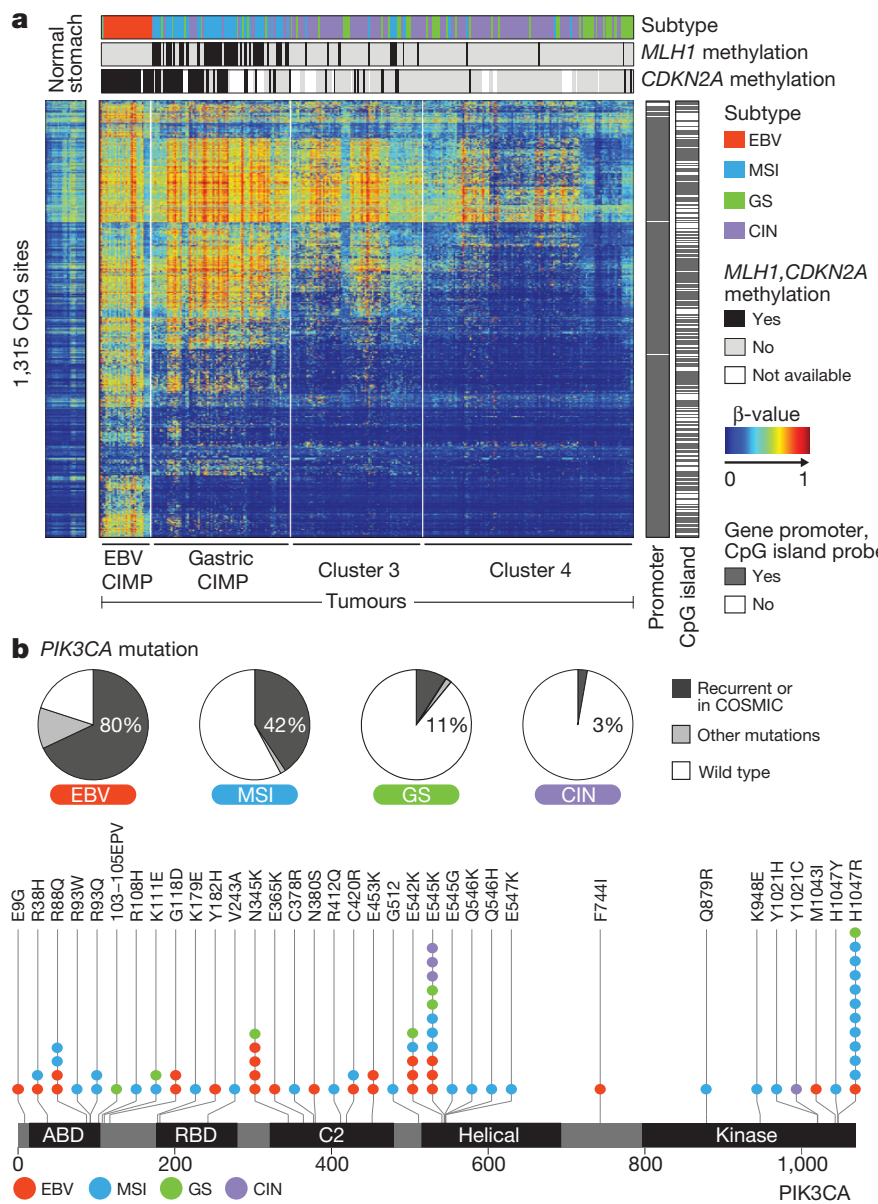

Figure $2 \mid$ Molecular characteristics of EBV-positive gastric cancers. a, The heatmap represents unsupervised clustering of DNA methylation at CpG sites for 295 tumours into four clusters: EBV-CIMP $(n=28)$, Gastric-CIMP $(n=77)$, cluster $3(n=73)$ and cluster $4(n=117)$. Profiles for non-malignant gastric mucosa are to the left of the tumours. $\mathbf{b}$, The proportion of tumours harbouring PIK3CA mutation in the molecular subtypes with mutations at sites noted recurrently in this data set or in the COSMIC database marked separately (top). Locations of PIK3CA mutations with the subtype of the sample with each mutation colour-coded (bottom).

complex class I genes, including B2M and $H L A-B$ (Supplementary Fig. 11.5-11.7). B2M mutations in colorectal cancers and melanoma result in loss of expression of HLA class 1 complexes ${ }^{23}$, suggesting these events benefit hypermutated tumours by reducing antigen presentation to the immune system.

Through MutSigCV analysis of the 215 non-hypermutated tumours, we identified 25 significantly mutated genes (Fig. 3). This gene list again included TP53, ARID1A, KRAS, PIK3CA and RNF43, but also genes in the $\beta$-catenin pathway ( $A P C$ and $C T N N B 1)$, the TGF- $\beta$ pathway (SMAD4 and SMAD2), and RASA1, a negative regulator of RAS. ERBB2, a therapeutic target, was significantly mutated, with 10 of 15 mutations occurring at known hotspots; four cases had the S310F ERBB2 mutation that is activating and drug-sensitive ${ }^{24}$

In addition to PIK3CA mutations, EBV-positive tumours had frequent ARID1A (55\%) and BCOR (23\%) mutations and only rare TP53 mutations. $B C O R$, encoding an anti-apoptotic protein, is also mutated in leukaemia ${ }^{25}$ and medulloblastoma ${ }^{26}$. Among the CIN tumours, we observed TP53 mutations in $71 \%$ of tumours. $\mathrm{CDH} 1$ somatic mutations were enriched in the genomically stable subtype (37\% of cases). $\mathrm{CDH} 1$ germline mutations underlie hereditary diffuse gastric cancer (HDGC). However, germline analysis revealed only two $C D H 1$ polymorphisms, neither of which is known to be pathogenic. As in the EBV-subtype, inactivating ARID1A mutations were prevalent in the genomically stable

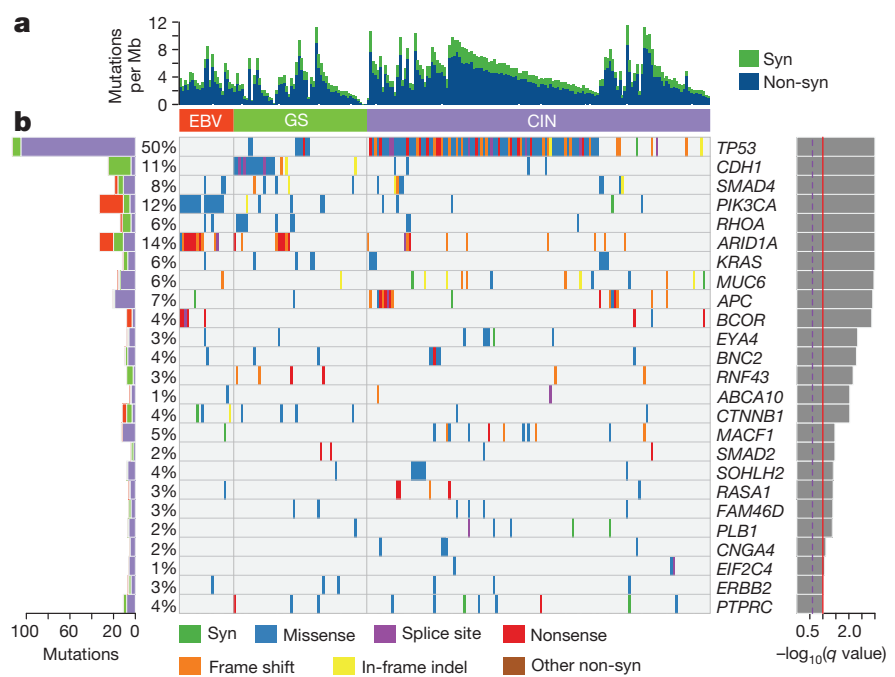

Figure 3 | Significantly mutated genes in non-hypermutated gastric cancer. a, Bars represent somatic mutation rate for the 215 samples with synonymous and non-synonymous mutation rates distinguished by colour. b, Significantly mutated genes, identified by MutSigCV, are ranked by the $q$ value (right) with samples grouped by subtype. Mutation colour indicates the class of mutation.

subtype. We identified mutations of RHOA almost exclusively in genomically stable tumours, as discussed below.

We analysed the patterns of base changes within gastric cancer tumours and noted elevated rates of $\mathrm{C}$ to $\mathrm{T}$ transitions at $\mathrm{CpG}$ dinucleotides. We observed an elevated rate of A to $\mathrm{C}$ transversions at the $3^{\prime}$ adenine of AA dinucleotides, especially at AAG trinucleotides, as reported in oesophageal adenocarcinoma ${ }^{27}$. The $\mathrm{A}$ to $\mathrm{C}$ transversions were prominent in CIN, EBV and genomically stable, but as previously observed ${ }^{27}$, not in MSI tumours (Supplementary Fig. 3.10).

We identified $R H O A$ mutation in 16 cases, and these were enriched in the genomically stable subtype ( $15 \%$ of genomically stable cases, $P=$ 0.0039). RHOA, when in the active GTP-bound form, acts through a variety of effectors, including ROCK1, mDIA and Protein Kinase N, to control actin-myosin-dependent cell contractility and cellular motility ${ }^{28,29}$

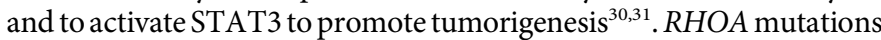
were clustered in two adjacent amino-terminal regions that are predicted to be at the interface of RHOA with ROCK1 and other effectors (Fig. 4a, b). RHOA mutations were not at sites analogous to oncogenic mutations in RAS-family GTPases. Although one case harboured a codon 17 mutation, we did not identify the dominant-negative G17V mutations noted in T-cell neoplasms ${ }^{32,33}$. Rather, the mutations found in this study may act to modulate signalling downstream of RHOA. Biochemical studies found that the RHOA Y42C mutation attenuated activation of Protein Kinase $\mathrm{N}$, without abrogated activation of $\mathrm{mDia}$ or ROCK $1^{34}$. RHOA Y42, mutated in five tumours, corresponds to Y40 on HRAS, a residue which when mutated selectively reduces HRAS activation of RAF, but not other RAS effectors ${ }^{35}$. Given the role of RHOA in cell motility, modulation of RHOA may contribute to the disparate growth patterns and lack of cellular cohesion that are hallmarks of diffuse tumours.

Dysregulated RHO signalling was further implicated by the discovery of recurrent structural genomic alterations. Whole genome sequencing of 107 tumours revealed 5,696 structural rearrangements, including 74 predicted to produce in-frame gene fusions (Supplementary Information S3.7-3.8). De novo assembly of mRNA sequencing data confirmed 170 structural rearrangements (Supplementary Information S5.4a), including two cases with an interchromosomal translocation between CLDN18 and ARHGAP26 (GRAF). ARHGAP26 is a GTPase-activating protein (GAP) that facilitates conversion of RHO GTPases to the GDP state and has been implicated in enhancing cellular motility ${ }^{34}$. CLDN18 


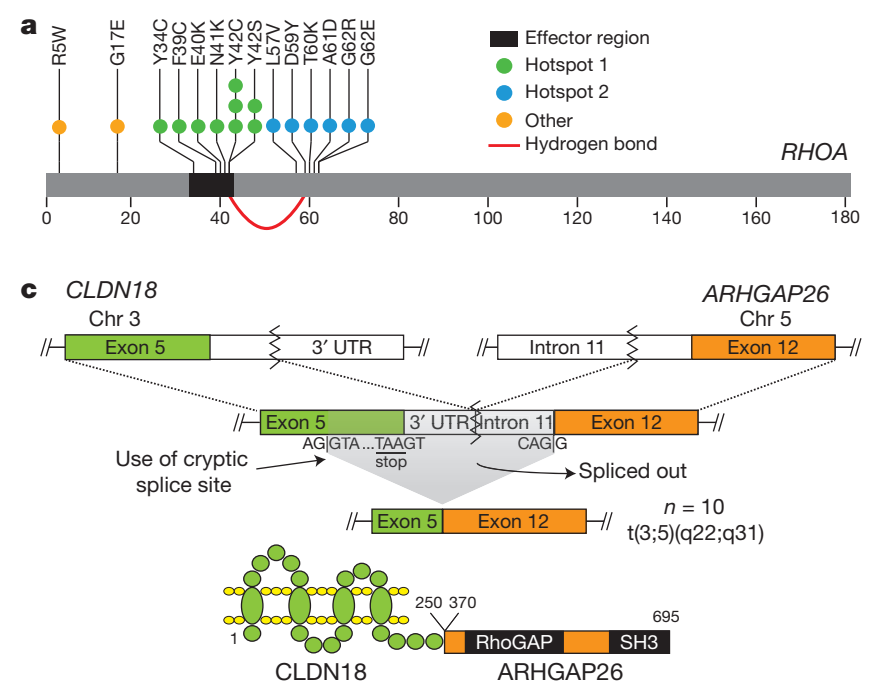

Figure $4 \mid R H O A$ and $A R H G A P 6 / 26$ somatic genomic alterations are recurrent in genomically stable gastric cancer. a, Missense mutations in the GTPase RHOA, including residues Y42 and D59, linked via hydrogen bond (red arc). b, Mutated regions (coloured as in panel a) mapped on the structures of RHOA and ROCK1.c, A schematic of CLDN18-ARHGAP26 translocation is

is a component of the tight junction adhesion structures ${ }^{36}$. RNA sequencing data from tumours without whole genome sequencing identified CLDN18-ARHGAP26 fusions in 9 additional tumours, with two more cases showing CLDN18 fusion to the homologous GAP encoded by ARHGAP6 totalling 13 cases with these rearrangements (Supplementary Table 5.6).

The fusions linked exon 5 of CLDN18 to exon $2(n=2)$ of ARHGAP6, to exon $10(n=1)$, or to exon $12(n=10)$ of ARHGAP26 (Fig. $4 \mathrm{c})$. As these fusions occur downstream of the CLDN18 exon 5 stop codon, they appeared unlikely to enable translation of fusion proteins. However, mRNA sequencing revealed a mature fusion transcript in which the ARHGAP26 or ARHGAP6 splice acceptor activates a cryptic splice site within exon 5 of $C L D N 18$, before the stop codon, yielding an inframe fusion predicted to maintain the transmembrane domains of CLDN18 while fusing a large segment of ARHGAP26 or ARHGAP6 to the cytoplasmic carboxy terminus of CLDN18. These chimaeric proteins retain the carboxy-terminal GAP domain of ARHGAP26/6, potentially affecting ARHGAP's regulation of RHOA and/or cell motility. Furthermore, these fusions may also disrupt wild-type CLDN18, impacting cellular adhesion. The CLDN18-ARHGAP fusions were mutually exclusive with $R H O A$ mutations and were enriched in genomically stable tumours $\left(62 \%, P=10^{-3}\right)$ (Fig. $4 \mathrm{~d}$ ). Within the genomically stable subtype, $30 \%$ of cases had either RHOA or CLDN18-ARHGAP alterations. Evaluation of gene expression status in pathways putatively regulated by RHOA using the Paradigm-Shift algorithm predicted activation of RHOA-driven pathways (Supplementary Fig. 11.4a-c), suggesting that these genomic aberrations contribute to the invasive phenotype of diffuse gastric cancer.

SCNA analysis using GISTIC identified 30 focal amplifications, 45 focal deletions, and chromosome arms subject to frequent alteration (Supplementary Figs 2.3-2.9). Focal amplifications targeted oncogenes such as ERBB2, CCNE1, KRAS, MYC, EGFR, CDK6, GATA4, GATA6 and $Z N F 217$. Additionally, we saw amplification of the gene that encodes the gastric stem cell marker CD44 and a novel recurrent amplification at 9p24.1 at the locus containing JAK2, CD274 and PDCD1LG2. JAK2 encodes a receptor tyrosine kinase and potential therapeutic target. CD274 and PDCD1LG2 encode PD-L1 and PD-L2, immunosuppressant proteins currently being evaluated as targets to augment anti-tumour immune response. Notably, these $9 p$ amplifications were enriched in the EBV subgroup (15\% of tumours), consistent with studies showing
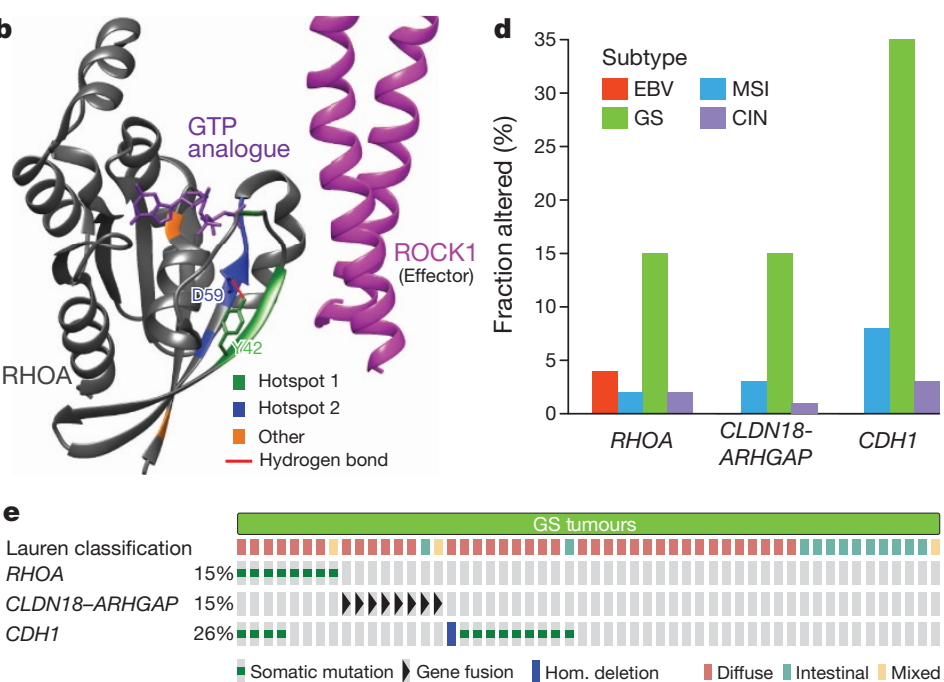

shown for the fusion transcript and predicted fusion protein. SH3 denotes SRC homology 3 domain. d, The frequency of $R H O A$ and $C D H 1$ mutations, CLDN18-ARHGAP6 or ARHGAP26 fusions are shown across gastric cancer subtypes. e, RHOA mutations and CLDN18-ARHGAP6 or ARHGAP26 fusions are mutually exclusive in genomically stable tumours.

elevated PD-L1 expression in EBV-positive lymphoid cancers ${ }^{37,38}$. Evaluation of mRNA revealed elevated expression of $J A K 2, P D-L 1$ and $P D$ L2 in amplified cases (Supplementary Fig. 2.10). More broadly, PD-L1/2 expression was elevated in EBV-positive tumours, suggesting that PDL1/2 antagonists and JAK2 inhibitors be tested in this subgroup. Focal deletions were identified at the loci of tumour suppressors such as PTEN, SMAD4, CDKN2A and ARID1A. Additional GISTIC analysis on the four molecular subtypes is detailed in Supplementary Figs 2.5-2.6.

\section{Gene expression and proteomic analysis}

Our analysis of each of the expression platforms revealed four mRNA, five miRNA and three RPPA clusters (Supplementary Methods S5-S7). Some expression clusters are similar across platforms (Supplementary Methods S10) and/or have correspondence with specific molecular subtypes. For example, mRNA cluster 1, miRNA cluster 4 and RPPA cluster 1 have substantial overlap and are strongly associated with genomically stable tumours, both individually and as a group; the 34 cases with all three assignments were predominantly genomically stable (20/ $\left.34, P=2 \times 10^{-8}\right)$. Similarly, mRNA cluster 3 , miRNA cluster 2 and RPPA cluster 3 are similar and are associated with the MSI subtype as a group $\left(12 / 22, P=5 \times 10^{-4}\right)$. However, absolute correspondence between expression clusters and molecular subtypes was not always seen. For example, RPPA cluster 3 showed moderate association with both MSI and EBV $(P=0.018$ and $P=0.038$, respectively), and miRNA clusters each had similar proportions of CIN (no associations with $P<$ 0.05). Overall, the expression data recapitulate features of the molecular classification, pointing to robustness of this taxonomy.

We analysed mRNA sequence data for alternative splicing events, finding MET exon 2 skipping in 82 of 272 (30\%) cases, associated with increased MET expression $\left(P=10^{-4}\right)$. We also found novel variants of $M E T$ in which exons 18 and/or 19 were skipped (47/272; 17\%; Supplementary Fig. 5.5). Intriguingly, the exons removed by these alterations encode regions of the kinase domain.

Through supervised analysis of RPPA data, we observed 45 proteins whose expression or phosphorylation was associated with the four molecular subtypes (Supplementary Fig. 7.2). Phosphorylation of EGFR (pY1068) was significantly elevated in the CIN subtype, consistent with amplification of EGFR within that subtype. We also found elevated expression of $\mathrm{p} 53$, consistent with frequent TP53 mutation and aneuploidy in the CIN subtype. 
a

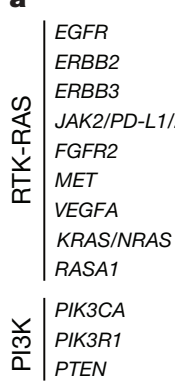

RHOA mutation CLDN18-ARHGAP Fusion)

b

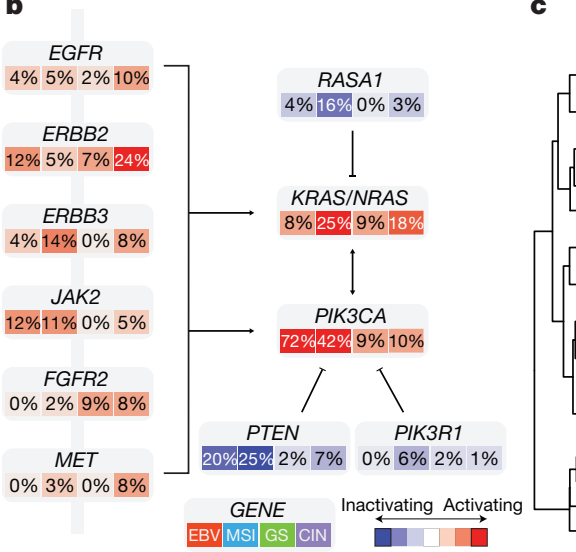

c
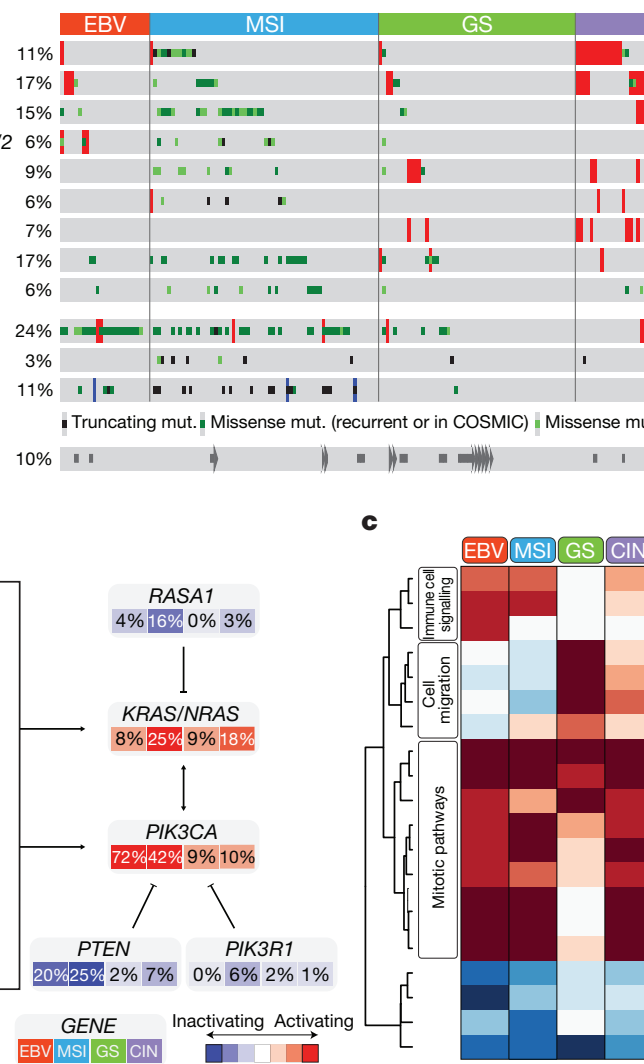
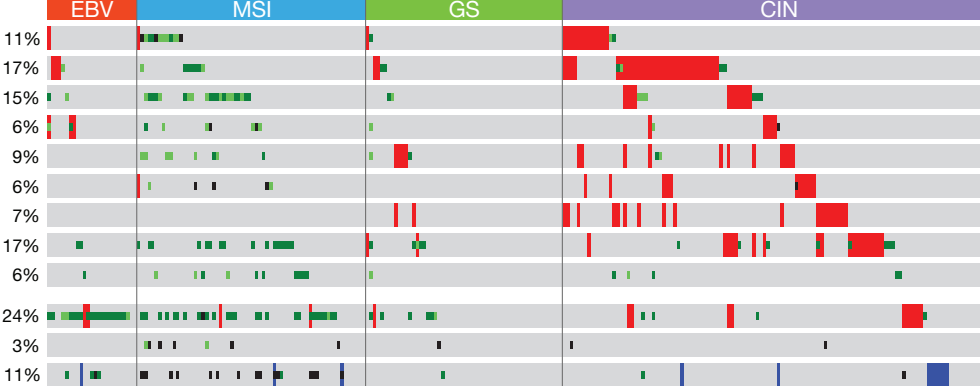

| Truncating mut.| Missense mut. (recurrent or in COSMIC) i| Missense mut. (all other) |Amplification | Hom. deletion

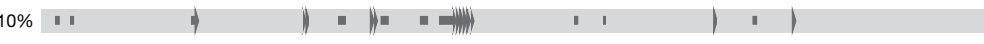

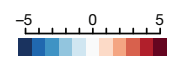

RANBP2-mediated transcriptional repression Caspase cascade in apoptosis L12-mediated signalling events Integrins in angiogenesis Syndecan-1-mediated signalling Beta1/Beta3 integrin cell surface interactions VEGFR3 signalling in lymphatic endothelium Fanconi anaemia pathway ATM/ATR/BARD1 TCPTP Signalling TCPTP Signalling p53 pathway

Regulation of retinoblastoma protein

Aurora A/B signalling FOXM1/PLK1 signalling E2F/targets of cMyc activation E2F/targets of cMyc activatic BMP receptor signalling ARF6 trafficking events

FOXA2/FOXA3 transcription factor networks
Figure 5 Integrated molecular description of gastric cancer. a, Mutations, copy-number changes and translocations for select genes are shown across samples organized by molecular subtypes. Mutations that are recurrent in this data set or in the COSMIC repository are distinguished by colour. Alteration frequencies are expressed as a percentage of all cases. $\mathbf{b}$, Alterations in RTK/RAS and RTK/PI(3)K signalling pathways across molecular subtypes. Red denotes predicted activation; blue denotes predicted inactivation. c, The heatmap shows NCI-PID pathways that are significantly elevated (red) or decreased (blue) in each of the four subtypes as compared with non-malignant gastric mucosa.

\section{Integrated pathway analysis}

We integrated SCNA and mutation data to characterize genomic alterations in known signalling pathways, including candidate therapeutic targets (Fig. 5a, b). We focused on alterations in receptor tyrosine kinases (RTKs) and RAS and PI(3)-kinase signalling. EBV-positive tumours contained PIK3CA mutations and recurrent JAK2 and ERBB2 amplifications. Although MSI cases generally lacked targetable amplifications, mutations in PIK3CA, ERBB3, ERBB2 and EGFR were noted, with many mutations at 'hotspot' sites seen in other cancers (Supplementary Fig. 11.14). Absent from MSI gastric cancers were BRAF V600E mutations, commonly seen in MSI colorectal cancer $^{39}$. Although the genomically stable subtype exhibited recurrent RHOA and CLDN18 events, few other clear treatment targets were observed. In CIN tumours, we identified genomic amplifications of RTKs, many of which are amenable to blockade by therapeutics in current use or in development. Recurrent amplification of the gene encoding ligand VEGFA was notable given the gastric cancer activity of the VEGFR2 targeting antibody ramucirumab $^{40}$. Additionally, frequent amplifications of cell cycle mediators (CCNE1, CCND1 and CDK6) suggest the potential for therapeutic inhibition of cyclin-dependent kinases (Supplementary Fig. 11.15).

We compared expression within each subtype to that of the other subtypes, and to non-malignant gastric tissue $(n=29)$ (Supplementary Fig. 11.2). We computed an aggregate score for each pathway of the NCI pathway interaction database ${ }^{41}$ and determined statistical significance by comparison with randomly generated pathways (Supplementary Methods S11). Hierarchical clustering of samples and pathways (Fig. 5c) revealed several notable patterns, including elevated expression of mitotic network components such as AURKA/B and E2F, targets of MYC activation, FOXM1 and PLK1 signalling and DNA damage response pathways across all subtypes, but to a lesser degree in genomically stable tumours. In contrast, the genomically stable subtype exhibited elevated expression of cell adhesion pathways, including the B1/B3 integrins, syndecan-1 mediated signalling, and angiogenesisrelated pathways. These results suggest additional candidate therapeutic targets, including the aurora kinases (AURKA/B) and Polo-like (PLK) family members. The strength of IL-12 mediated signalling signatures in EBV-positive tumours suggests a robust immune cell presence. When coupled with evidence of PD-L1/2 overexpression, this finding adds rationale for testing immune checkpoint inhibitors in EBV-positive gastric cancer.

\section{Discussion}

Through this study of the molecular and genomic basis of gastric cancer, we describe a molecular classification (Fig. 6) that defines four major genomic subtypes of gastric cancer: EBV-infected tumours; MSI tumours; genomically stable tumours; and chromosomally unstable tumours. This

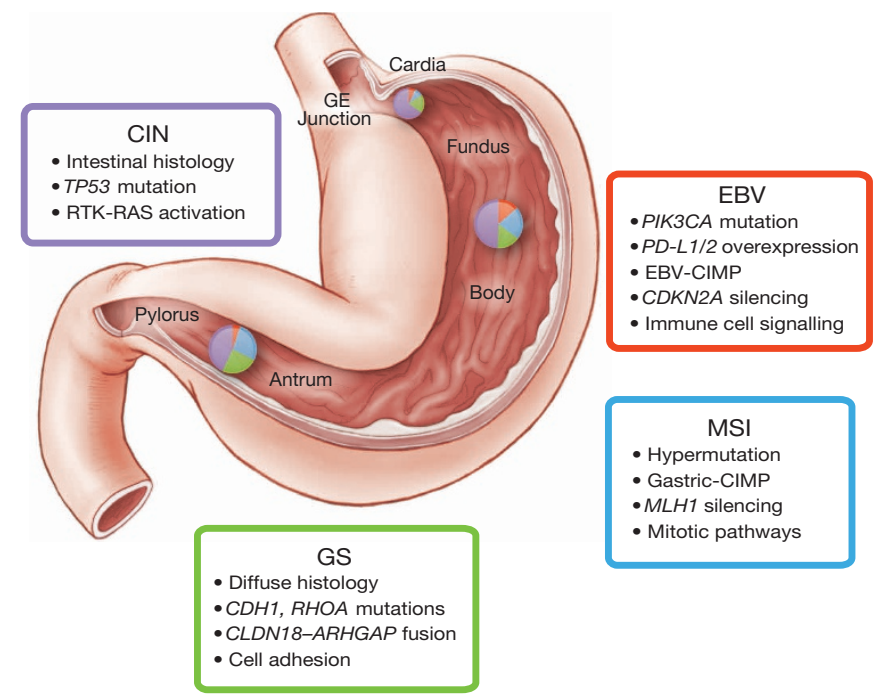

Figure $6 \mid$ Key features of gastric cancer subtypes. This schematic lists some of the salient features associated with each of the four molecular subtypes of gastric cancer. Distribution of molecular subtypes in tumours obtained from distinct regions of the stomach is represented by inset charts. 
classification may serve as a valuable adjunct to histopathology. Importantly, these molecular subtypes showed distinct salient genomic features, providing a guide to targeted agents that should be evaluated in clinical trials for distinct populations of gastric cancer patients. Through existing testing for MSI and EBV and the use of emerging genomic assays that query focused gene sets for mutations and amplifications, the classification system developed through this study can be applied to new gastric cancer cases. We hope these results will facilitate the development of clinical trials to explore therapies in defined sets of patients, ultimately improving survival from this deadly disease.

\section{METHODS SUMMARY}

Fresh frozen gastric adenocarcinoma and matched germline DNA samples were obtained from 295 patients under IRB approved protocols. Genomic material and (when available) protein were subjected to single nucleotide polymorphism array somatic copy-number analysis, whole-exome sequencing, mRNA sequencing, miRNA sequencing, array-based DNA methylation profiling and reverse-phase protein arrays. A subset of samples was subjected to whole-genome sequencing. Initial analysis centred on the development of a classification scheme for gastric cancer. Subsequent analysis identified key features from each of the genomic/molecular platforms, looking both for features found across gastric cancer and those characteristic of individual gastric cancer subtypes. Primary and processed data are deposited at the Data Coordinating Center (https://tcga-data.nci.nih.gov/tcga/tcgaDownload. jsp); primary sequence files are deposited in CGHub (https://cghub.ucsc.edu/). Sample lists, and supporting data can be found at (https://tcga-data.nci.nih.gov/ docs/publications/stad_2014/).

\section{Received 21 February; accepted 13 May 2014. \\ Published online 23 July; corrected online 10 September 2014 (see full-text HTML version for details).}

1. Ferlay, J. et al. GLOBOCAN 2012 v1.0, cancer incidence and mortality worldwide. IARC CancerBase 11, http://globocan.iarc.fr, accessed on January 15, 2014 (2013).

2. Laurén, $P$. The two histological main types of gastric carcinoma: diffuse and socalled intestinal-type carcinoma. Acta Pathol., Microbiol. Scand. 64, 31-49 (1965)

3. WHO Classification of Tumours of the Digestive System 4th edn (International Agency for Research on Cancer, 2010).

4. Uemura, N. et al. Helicobacter pylori infection and the development of gastric cancer. N. Engl. J. Med. 345, 784-789 (2001).

5. Bertuccio, P. et al. Recent patterns in gastric cancer: a global overview. International J. Cancer 125, 666-673 (2009).

6. Richards, F. M. et al. Germline E-cadherin gene ( $\mathrm{CDH} 1)$ mutations predispose to familial gastric cancer and colorectal cancer. Hum. Mol. Genet. 8, 607-610 (1999).

7. Keller, G. et al. Analysis for microsatellite instability and mutations of the DNA mismatch repair gene hMLH1 in familial gastric cancer. International J Cancer 68 571-576 (1996).

8. Toyota, M. et al. Aberrant methylation in gastric cancer associated with the CpG island methylator phenotype. Cancer Res. 59, 5438-5442 (1999).

9. Tan, I. B. et al. Intrinsic subtypes of gastric cancer, based on gene expression pattern, predict survival and respond differently to chemotherapy. Gastroenterology 141, 476-485 (2011)

10. Lei, Z. et al. Identification of molecular subtypes of gastric cancer with different responses to PI3-kinase inhibitors and 5-fluorouracil. Gastroenterology 145, 554-565 (2013).

11. Boussioutas, A. et al. Distinctive patterns of gene expression in premalignant gastric mucosa and gastric cancer. Cancer Res. 63, 2569-2577 (2003).

12. Wang, K. et al. Exome sequencing identifies frequent mutation of ARID1A in molecular subtypes of gastric cancer. Nature Genet. 43, 1219-1223 (2011).

13. Shen, R., Olshen, A. B. \& Ladanyi, M. Integrative clustering of multiple genomic data types using a joint latent variable model with application to breast and lung cancer subtype analysis. Bioinformatics 25, 2906-2912 (2009).

14. Murphy, G., Pfeiffer, R., Camargo, M. C. \& Rabkin, C. S. Meta-analysis shows that prevalence of Epstein-Barr virus-positive gastric cancer differs based on sex and anatomic location. Gastroenterology 137, 824-833 (2009).

15. Matsusaka, K. et al. Classification of Epstein-Barr virus-positive gastric cancers by definition of DNA methylation epigenotypes. Cancer Res. 71, 7187-7197 (2011).

16. Geddert, H.,Zur Hausen, A., Gabbert, H. E. \& Sarbia, M. EBV-infection in cardiac and non-cardiac gastric adenocarcinomas is associated with promoter methylation of p16, p14 and APC, but not hMLH1. Anal. Cell. Pathol. 33, 143-149 (2010).

17. Lee, J. et al. High-throughput mutation profiling identifies frequent somatic mutations in advanced gastric adenocarcinoma. PLOS ONE 7, e38892 (2012).

18. Sukawa, $Y$. et al. Alterations in the human epidermal growth factor receptor 2-phosphatidylinositol 3-kinase-v-Akt pathway in gastric cancer. World J. Gastroenterology 18, 6577-6586 (2012)

19. Strong, M. J. et al. Differences in gastric carcinoma microenvironment stratify according to EBV infection intensity: implications for possible immune adjuvant therapy. PLoS Pathog. 9, e1003341 (2013).
20. The Cancer Genome Atlas Network Comprehensive molecular characterization of human colon and rectal cancer. Nature 487, 330-337 (2012)

21. Cancer Genome Atlas Research Network. Integrated genomic characterization of endometrial carcinoma. Nature 497, 67-73 (2013).

22. Lawrence, M. S. et al. Mutational heterogeneity in cancer and the search for new cancer-associated genes. Nature 499, 214-218 (2013)

23. Bernal, M., Ruiz-Cabello, F., Concha, A., Paschen, A. \& Garrido, F. Implication of the $\beta 2$-microglobulin gene in the generation of tumor escape phenotypes. Cancer Immunol. Immunother. 61, 1359-1371 (2012).

24. Greulich, $\mathrm{H}$. et al. Functional analysis of receptor tyrosine kinase mutations in lung cancer identifies oncogenic extracellular domain mutations of ERBB2. Proc. Natl Acad. Sci. USA 109, 14476-14481 (2012)

25. Grossmann, V. et al. Whole-exome sequencing identifies somatic mutations of BCOR in acute myeloid leukemia with normal karyotype. Blood 118, 6153-6163 (2011).

26. Pugh, T. J. et al. Medulloblastoma exome sequencing uncovers subtype-specific somatic mutations. Nature 488, 106-110 (2012).

27. Dulak, A. M. et al. Exome and whole-genome sequencing of esophageal adenocarcinoma identifies recurrent driver events and mutational complexity. Nature Genet. 45, 478-486 (2013).

28. Ridley, A. J. et al. Cell migration: integrating signals from front to back. Science $\mathbf{3 0 2}$ 1704-1709 (2003)

29. Thumkeo, D., Watanabe, S. \& Narumiya, S. Physiological roles of Rho and Rho effectors in mammals. Eur. J. Cell Biol. 92, 303-315 (2013).

30. Aznar, S. et al. Simultaneous tyrosine and serine phosphorylation of STAT3 transcription factor is involved in Rho A GTPase oncogenic transformation. Mol. Biol. Cell 12, 3282-3294 (2001)

31. Yu, H. \& Jove, R. The STATs of cancer-new molecular targets come of age. Nature Rev. Cancer 4, 97-105 (2004)

32. Palomero, T. et al. Recurrent mutations in epigenetic regulators, RHOA and FYN kinase in peripheral T cell lymphomas. Nature Genet. 46, 166-170 (2014).

33. Sakata-Yanagimoto, M. et al. Somatic RHOA mutation in angioimmunoblastic T cell lymphoma. Nature Genet. 46, 171-175 (2014).

34. Doherty, G. J. et al. The endocytic protein GRAF1 is directed to cell-matrix adhesion sites and regulates cell spreading. Mol. Biol. Cell 22, 4380-4389 (2011).

35. Joneson, T., White, M. A., Wigler, M. H. \& Bar-Sagi, D. Stimulation of membrane ruffling and MAP kinase activation by distinct effectors of RAS. Science $\mathbf{2 7 1}$, 810-812 (1996).

36. Türeci, 0 . et al. Claudin-18 gene structure, regulation, and expression is evolutionary conserved in mammals. Gene 481, 83-92 (2011)

37. Chen, B. J. et al. PD-L1 expression is characteristic of a subset of aggressive B-cell lymphomas and virus-associated malignancies. Clinical Cancer Res 19, 3462-3473 (2013).

38. Green, M. R. et al. Constitutive AP-1 activity and EBV infection induce PD-L1 in Hodgkin lymphomas and posttransplant lymphoproliferative disorders: implications for targeted therapy. Clinical Cancer Res 18, 1611-1618 (2012).

39. The Cancer Genome Atlas Network Comprehensive molecular characterization of human colon and rectal cancer. Nature 487, 330-337 (2012).

40. Fuchs, C. S. et al. Ramucirumab monotherapy for previously treated advanced gastric or gastro-oesophageal junction adenocarcinoma (REGARD): an international, randomised, multicentre, placebo-controlled, phase 3 trial. Lancet 383, 31-39 (2014)

41. Schaefer, C. F. et al. PID: the pathway interaction database. Nucleic Acids Res. 37, D674-D679 (2009)

Supplementary Information is available in the online version of the paper.

Acknowledgements We are grateful to all the patients and families who contributed to this study and to C. Gunter and J. Weinstein for scientific editing, to M. Sheth for administrative support and to L. Omberg for support with the Sage Bionetworks Synapse platform. This work was supported by the Intramural Research Program and the following grants from the United States National Institutes of Health: 5U24CA143799, 5U24CA143835, 5U24CA143840, 5U24CA143843, 5U24CA143845, 5U24CA143848, 5U24CA143858, 5U24CA143866, 5U24CA143867, 5U24CA143882, 5U24CA143883, 5U24CA144025, U54HG003067, U54HG003079, U54HG003273 and P30CA16672.

Author Contributions The Cancer Genome Atlas Research Network contributed collectively to this study. Biospecimens were provided by the tissue source sites and processed by the Biospecimen Core Resource. Data generation and analyses were performed by the genome-sequencing centres, cancer genome-characterization centres and genome data analysis centres. All data were released through the Data Coordinating Center. The $\mathrm{NCl}$ and NHGRI project teams coordinated project activities. The following TCGA investigators of the Stomach Analysis Working Group contributed substantially to the analysis and writing of this manuscript. Project leaders, A. J. Bass, P. W. Laird, I. Shmulevich; data coordinator, V. Thorsson; analysis coordinators, V. Thorsson, N. Schultz; manuscript coordinator, M. Sheth; graphics coordinator, T. Hinoue; DNA sequence analysis, A. Taylor-Weiner, A. Pantazi, C. Sougnez, K. Kasaian; mRNA analysis, R. Bowlby, A. J. Mungall; miRNA analysis, A. Chu, A. Gordon Robertson, D. Yang; DNA methylation analysis, T. Hinoue, H. Shen, P. W. Laird; copy number analysis, A. Cherniack; protein analysis, J.-S. Lee, R. Akbani; pathway/integrated analysis, N. Weinhold, S. Reynolds, C. Curtis, R. Shen, S. Ng, B. Raphael, H.-T. Wu, Y. Liu, V. Thorsson, N. Schultz; pathology expertise and clinical data, A. Boussioutas, B. G. Schneider, J. Kim, J. E. Willis, M. L. Gulley, K. Garman, M. Blanca Piazuelo, V. Thorsson, K M. Leraas, T. Lichtenberg, J. A. Demchok, A. J. Bass; microbiome analysis, C. S. Rabkin, M. L. Gulley, R. Bowlby, A. J. Mungall, A. Chu and C. Pedamallu. 
Author Information The primary and processed data used to generate the analyses presented here can be downloaded from The Cancer Genome Atlas at (https:// tcga-data.nci.nih.gov/tcga/tcgaDownload.jsp). All of the primary sequence files are deposited in CGHub and all other data are deposited at the Data Coordinating Center (DCC) for public access (http://cancergenome.nih.gov/) and (https://cghub.ucsc. edu/). Additional sample data and supporting data are available from (https:// tcga-data.nci.nih.gov/docs/publications/stad_2014/). Reprints and permissions information is available at www.nature.com/reprints. The authors declare no competing financial interests. Readers are welcome to comment on the online version of the paper. Correspondence and requests for materials should be addressed to A.J.B. (adam_bass@dfci.harvard.edu).

This work is licensed under a Creative Commons AttributionNonCommercial-ShareAlike 3.0 Unported licence. The images or other third party material in this article are included in the article's Creative Commons licence, unless indicated otherwise in the credit line; if the material is not included under the Creative Commons licence, users will need to obtain permission from the licence holder to reproduce the material. To view a copy of this licence, visit http://creativecommons. org/licenses/by-nc-sa/3.0

\section{The Cancer Genome Atlas Research Network}

Analysis Working Group: Dana-Farber Cancer Institute Adam J. Bass ${ }^{1}$; Institute for Systems Biology Vesteinn Thorsson ${ }^{2}$, llya Shmulevich², Sheila M. Reynolds ${ }^{2}$, Michae Miller $^{2}$, Brady Bernard ${ }^{2}$; University of Southern California Toshinori Hinoue ${ }^{3}$, Peter W. Laird $^{3}$, Christina Curtis ${ }^{4}$, Hui Shen ${ }^{3}$, Daniel J. Weisenberger ${ }^{3}$; Memorial Sloan Kettering Cancer Center Nikolaus Schultz ${ }^{5}$, Ronglai Shen ${ }^{6}$, Nils Weinhold ${ }^{5}$, David P. Kelsen ${ }^{7}$; BC Cancer Agency Reanne Bowlby ${ }^{8}$, Andy Chu ${ }^{8}$, Katayoon Kasaian ${ }^{8}$, Andrew J. Mungall ${ }^{8}$, A. Gordon Robertson ${ }^{8}$, Payal Sipahimalani ${ }^{8}$; The Eli \& Edythe L. Broad Institute Andrew D. Cherniack ${ }^{9}$, Gad Getz ${ }^{9}$, Yingchun Liu ${ }^{9}$, Michael S. Noble ${ }^{9}$, Chandra Pedamallu ${ }^{9}$, Carrie Sougnez ${ }^{9}$, Amaro Taylor-Weiner ${ }^{9}$; MD Anderson Cancer Center Rehan Akbani ${ }^{10}$, Ju-Seog Lee ${ }^{10}$, Wenbin Liu ${ }^{10}$, Gordon B. Mills ${ }^{11}$, Da Yang ${ }^{12}$, Wei Zhang $^{12}$; Harvard Medical School Angeliki Pantazi ${ }^{13}$, Michael Parfenov ${ }^{13}$; University of North Carolina Margaret Gulley ${ }^{14}$; Vanderbilt University M. Blanca Piazuelo ${ }^{15}$, Barbara G. Schneider ${ }^{15}$; Asan Medical Center Jihun Kim ${ }^{16}$; University of Melbourne Alex Boussioutas ${ }^{17}$; National Cancer Institute Margi Sheth ${ }^{18}$, John A. Demchok ${ }^{18}$, Charles S. Rabkin ${ }^{19}$; Case Western Reserve University Joseph E. Willis ${ }^{20}$; University of California at Santa Cruz Sam Ng${ }^{21}$; Duke University Katherine Garman ${ }^{22}$; University of Michigan David G. Beer ${ }^{23}$; University of Pittsburgh Arjun Pennathur ${ }^{24}$; Brown University Benjamin J. Raphael ${ }^{25}$, Hsin-Ta Wu ${ }^{25}$; Brigham and Women's Hospital Robert Odze ${ }^{26}$; National Cancer Center Hark K. Kim² ${ }^{27}$; Nationwide Children's Hospital Jay Bowen ${ }^{28}$, Kristen M. Leraas ${ }^{28}$, Tara M. Lichtenberg ${ }^{28}$, Stephanie Weaver ${ }^{28}$; Washington University Michael McLellan ${ }^{29}$; Greater Poland Cancer Centre Maciej Wiznerowicz ${ }^{30}$, KU Leuven: Ryo Sakai ${ }^{31}$

Genome Sequencing Center: The Eli \& Edythe L. Broad Institute Gad Getz ${ }^{9}$, Carrie Sougnez ${ }^{9}$, Michael S. Lawrence ${ }^{9}$, Kristian Cibulskis ${ }^{9}$, Lee Lichtenstein ${ }^{9}$, Sheila Fisher ${ }^{9}$, Stacey B. Gabriel ${ }^{9}$, Eric S. Lander ${ }^{9}$; Washington University in St. Louis Li Ding ${ }^{29}$, Beifang Niu ${ }^{29}$

Genome Characterization Centers: BC Cancer Agency Adrian Ally ${ }^{8}$, Miruna Balasundaram ${ }^{8}$, Inanc Birol ${ }^{8}$, Reanne Bowlby ${ }^{8}$, Denise Brooks ${ }^{8}$, Yaron S. N. Butterfield $^{8}$, Rebecca Carlsen ${ }^{8}$, Andy $\mathrm{Chu}^{8}$, Justin $\mathrm{Chu}^{8}$, Eric Chuah ${ }^{8}$, Hye-Jung E. Chun ${ }^{8}$, Amanda Clarke ${ }^{8}$, Noreen Dhalla ${ }^{8}$, Ranabir Guin ${ }^{8}$, Robert A. Holt ${ }^{8}$, Steven J. M. Jones ${ }^{8}$, Katayoon Kasaian ${ }^{8}$, Darlene Lee ${ }^{8}$, Haiyan A. Li ${ }^{8}$, Emilia Lim ${ }^{8}$, Yussanne Ma ${ }^{8}$, Marco A. Marra ${ }^{8}$, Michael Mayo ${ }^{8}$, Richard A. Moore ${ }^{8}$, Andrew J. Mungall ${ }^{8}$, Karen L. Mungall ${ }^{8}$, Ka Ming Nip ${ }^{8}$, A. Gordon Robertson ${ }^{8}$, Jacqueline E. Schein ${ }^{8}$, Payal Sipahimalani ${ }^{8}$, Angela Tam ${ }^{8}$, Nina Thiessen ${ }^{8}$; The Eli \& Edythe L. Broad Institute Rameen Beroukhim ${ }^{9}$, Scott L. Carter ${ }^{9}$, Andrew D. Cherniack ${ }^{9}$, Juok Cho ${ }^{9}$, Kristian Cibulskis $^{9}$, Daniel DiCara ${ }^{9}$, Scott Frazer ${ }^{9}$, Sheila Fisher ${ }^{9}$, Stacey B. Gabriel ${ }^{9}$, Nils Gehlenborg' ${ }^{9}$, David I. Heiman ${ }^{9}$, Joonil Jung' ${ }^{9}$, Jaegil Kim', Eric S. Lander ${ }^{9}$, Michael S. Lawrence $^{9}$, Lee Lichtenstein ${ }^{9}$, Pei Lin ${ }^{9}$, Matthew Meyerson' ${ }^{9}$, Akinyemi I. Ojesina', Chandra Sekhar Pedamallu ${ }^{9}$, Gordon Saksena ${ }^{9}$, Steven E. Schumacher ${ }^{9}$, Carrie Sougnez ${ }^{9}$, Petar Stojanov ${ }^{9}$, Barbara Tabak ${ }^{9}$, Amaro Taylor-Weiner ${ }^{9}$, Doug Voet ${ }^{9}$, Mara Rosenberg', Travis I. Zack' ${ }^{9}$, Hailei Zhang', Lihua Zou'; Harvard Medical School/ Brigham \& Women's Hospital/MD Anderson Cancer Center Alexei Protopopov ${ }^{32}$ Netty Santoso ${ }^{13}$, Michael Parfenov ${ }^{13}$, Semin Lee ${ }^{33}$, Jianhua Zhang ${ }^{32}$, Harshad S. Mahadeshwar ${ }^{32}$, Jiabin Tang ${ }^{32}$, Xiaojia Ren ${ }^{13}$, Sahil Seth ${ }^{32}$, Lixing Yang ${ }^{33}$, Andrew W. $\mathrm{Xu}^{33}$, Xingzhi Song ${ }^{32}$, Angeliki Pantazi ${ }^{13}$, Ruibin Xi ${ }^{33}$, Christopher A. Bristow ${ }^{32}$, Angela Hadjipanayis ${ }^{13}$, Jonathan Seidman ${ }^{13}$, Lynda Chin ${ }^{32}$, Peter J. Park ${ }^{33}$, Raju Kucherlapati ${ }^{13}$; MD Anderson Cancer Center Rehan Akbani ${ }^{10}$, Shiyun Ling ${ }^{10}$, Wenbin $\mathrm{Liu}^{10}$, Arvind Rao ${ }^{10}$, John N. Weinstein ${ }^{10}$, Sang-Bae Kim ${ }^{11}$, Ju-Seog Lee ${ }^{11}$, Yiling Lu ${ }^{11}$ Gordon Mills ${ }^{11}$; University of Southern California Epigenome Center Peter W. Laird ${ }^{3}$ Toshinori Hinoue ${ }^{3}$, Daniel J. Weisenberger ${ }^{3}$, Moiz S. Bootwalla ${ }^{3}$, Phillip H. Lai ${ }^{3}$, Hui Shen $^{3}$, Timothy Triche Jr ${ }^{3}$, David J. Van Den Berg ${ }^{3}$; The Sidney Kimmel Comprehensive Cancer Center at Johns Hopkins University Stephen B. Baylin ${ }^{34}$ James G. Herman ${ }^{34}$

Genome Data Analysis Centers: The Eli \& Edythe L. Broad Institute Gad Getz ${ }^{9}$, Lynda Chin $^{32}$, Yingchun Liu' ${ }^{9}$, Bradley A. Murray ${ }^{9}$, Michael S. Noble ${ }^{9}$; Memorial Sloan-Kettering Cancer Center B. Arman Askoy ${ }^{5}$, Giovanni Ciriello ${ }^{5}$, Gideon Dresdner ${ }^{5}$ Jianjiong Gao ${ }^{5}$, Benjamin Gross ${ }^{5}$, Anders Jacobsen ${ }^{5}$, William Lee ${ }^{5}$, Ricardo Ramirez ${ }^{5}$,
Chris Sander ${ }^{5}$, Nikolaus Schultz ${ }^{5}$, Yasin Senbabaoglu ${ }^{5}$, Rileen Sinha ${ }^{5}$, S. Onur Sumer ${ }^{5}$, Yichao Sun ${ }^{5}$, Nils Weinhold ${ }^{5}$; Institute for Systems Biology Vésteinn Thorsson ${ }^{2}$, Brady Bernard $^{2}$, Lisa lype ${ }^{2}$, Roger W. Kramer², Richard Kreisberg ${ }^{2}$, Michael Miller ${ }^{2}$, Sheila M. Reynolds ${ }^{2}$, Hector Rovira ${ }^{2}$, Natalie Tasman ${ }^{2}$, Ilya Shmulevich ${ }^{2}$; University of California, Santa Cruz Sam Ng${ }^{21}$, David Haussler ${ }^{21}$, Josh M. Stuart ${ }^{21}$; MD Anderson Cancer Center Rehan Akbani ${ }^{10}$, Shiyun Ling ${ }^{10}$, Wenbin Liu ${ }^{10}$, Arvind Rao ${ }^{10}$, John N. Weinstein ${ }^{10}$, Roeland G. W. Verhaak ${ }^{32}$, Gordon B. Mills ${ }^{11}$; Brown University Mark D. M Leiserson ${ }^{25}$, Benjamin J. Raphael ${ }^{25}$, Hsin-Ta Wu ${ }^{25}$; University of California San Francisco Barry S. Taylor ${ }^{35}$

Biospecimen Core Resource: The Research Institute at Nationwide Children's Hospital Aaron D. Black ${ }^{28}$, Jay Bowen ${ }^{28}$, Julie Ann Carney ${ }^{28}$, Julie M. Gastier-Foster ${ }^{28}$ Carmen Helsel $^{28}$, Kristen M. Leraas ${ }^{28}$, Tara M. Lichtenberg ${ }^{28}$, Cynthia McAllister ${ }^{28}$, Nilsa C. Ramirez ${ }^{28}$, Teresa R. Tabler ${ }^{28}$, Lisa Wise ${ }^{28}$, Erik Zmuda ${ }^{28}$; International Genomics Consortium Robert Penny ${ }^{36}$, Daniel Crain ${ }^{36}$, Johanna Gardner ${ }^{36}$, Kevin Lau $^{36}$, Erin Curely ${ }^{36}$, David Mallery ${ }^{36}$, Scott Morris ${ }^{36}$, Joseph Paulauskis ${ }^{36}$, Troy Shelton $^{36}$, Candace Shelton ${ }^{36}$, Mark Sherman ${ }^{36}$

Tissue Source Sites: Buck Institute for Research on Aging Christopher Benz ${ }^{37}$; Chonnam National University Medical School Jae-Hyuk Lee ${ }^{38}$; City Clinical Oncology Dispensary Konstantin Fedosenko ${ }^{39}$, Georgy Manikhas ${ }^{39}$; Cureline Olga Potapova ${ }^{40}$ Olga Voronina ${ }^{40}$, Dmitry Belyaev ${ }^{40}$, Oleg Dolzhansky ${ }^{40}$; UNC Lineberger Comprehensive Cancer Center W. Kimryn Rathmell ${ }^{41}$; 'Greater Poland Cancer Centre Jakub Brzezinski ${ }^{30}$, Matthew Ibbs $^{30}$, Konstanty Korski ${ }^{30}$, Witold Kycler ${ }^{30}$, Radoslaw Łaźniak $^{30}$, Ewa Leporowska ${ }^{30}$, Andrzej Mackiewicz ${ }^{30}$, Dawid Murawa ${ }^{30}$, Pawel Murawa ${ }^{30}$, Arkadiusz Spychała ${ }^{30}$, Wiktoria M. Suchorska ${ }^{30}$, Honorata Tatka ${ }^{30}$, Marek Teresiak $^{30}$, Maciej Wiznerowicz ${ }^{30}$; Helen F. Graham Cancer Center \& Research Institute Raafat Abdel-Misih ${ }^{42}$, Joseph Bennett ${ }^{42}$, Jennifer Brown ${ }^{42}$, Mary lacocca ${ }^{42}$ Brenda Rabeno ${ }^{42}$; Keimyung University School of Medicine Sun-Young Kwon ${ }^{43}$; International Genomics Consortium Robert Penny ${ }^{36}$, Johanna Gardner ${ }^{36}$, Ariane Kemkes $^{36}$, David Mallery ${ }^{36}$, Scott Morris ${ }^{36}$, Troy Shelton ${ }^{36}$, Candace Shelton ${ }^{36}$, Erin Curley $^{36}$; Ontario Tumour Bank lakovina Alexopoulou ${ }^{44}$, Jay Engel ${ }^{45}$, John Bartlett ${ }^{46}$ Monique Albert ${ }^{46}$; Pusan National University Hospital Do-Youn Park ${ }^{47}$; University of Pittsburgh School of Medicine Rajiv Dhir ${ }^{24}$, James Luketich ${ }^{24}$, Rodney Landreneau ${ }^{24}$

Disease Working Group: Memorial Sloan-Kettering Cancer Center Yelena Y. Janjigian ${ }^{7}$, David P. Kelsen ${ }^{7}$, Eunjung Cho ${ }^{48}$, Marc Ladanyi ${ }^{48}$, Laura Tang ${ }^{48}$; Duke University Shannon J. McCall ${ }^{49}$; Asan Medical Center Young S. Park ${ }^{16}$; Yonsei University College of Medicine Jae-Ho Cheong ${ }^{50}$; MD Anderson Cancer Center Jaffer Ajani $^{51}$; National Cancer Institute M. Constanza Camargo ${ }^{19}$

Data Coordination Center: SRA International Shelley Alonso ${ }^{52}$, Brenda Ayala ${ }^{52}$, Mark A. Jensen ${ }^{52}$, Todd Pihl ${ }^{52}$, Rohini Raman ${ }^{52}$, Jessica Walton ${ }^{52}$, Yunhu Wan ${ }^{52}$

Project Team: National Cancer Institute John A. Demchok ${ }^{18}$, Greg Eley ${ }^{18}$, Kenna R. Mills Shaw ${ }^{18}$, Margi Sheth ${ }^{18}$, Roy Tarnuzzer ${ }^{18}$, Zhining Wang ${ }^{18}$, Liming Yang ${ }^{18}$, Jean Claude Zenklusen ${ }^{18}$, Tanja Davidsen ${ }^{53}$, Carolyn M. Hutter ${ }^{54}$, Heidi J. Sofia ${ }^{54}$; SAIC-Frederick Robert Burton ${ }^{55}$, Sudha Chudamani ${ }^{55}$ \& Jia Liu ${ }^{55}$

${ }^{1}$ Department of Medical Oncology and the Center for Cancer Genome Discovery, Dana-Farber Cancer Institute, Boston, Massachusetts 02215, USA. ${ }^{2}$ Institute for Systems Biology, Seattle, Washington 98109, USA. ${ }^{3}$ USC Epigenome Center, University of Southern California, Los Angeles, California 90033, USA. ${ }^{4}$ University of Southern California, Department of Preventive Medicine, USC/Norris Comprehensive Cancer Center, Los Angeles, California 90033, USA. ${ }^{5}$ Computational Biology Center, Memorial Sloan-Kettering Cancer Center, New York, New York 10065, USA. ${ }^{6}$ Department of Epidemiology and Biostatistics, Memorial Sloan-Kettering Cancer Center, New York, New York 10065, USA. ${ }^{7}$ Department of Medicine, Memorial Sloan-Kettering Cancer Center, 1275 York Avenue, New York, New York 10021, USA. ${ }^{8}$ Canada's Michael Smith Genome Sciences Centre, BC Cancer Agency, Vancouver, BC V5Z 4S6, Canada. ${ }^{9}$ The Eli and Edythe L. Broad Institute, Cambridge, Massachusetts 02142, USA. ${ }^{10}$ Department of Bioinformatics and Computational Biology, University of Texas MD Anderson Cancer Center, Houston, Texas 77030, USA. ${ }^{11}$ Department of Systems Biology, University of Texas MD Anderson Cancer Center, Houston, Texas 77030, USA. ${ }^{12}$ Department of Pathology, University of Texas MD Anderson Cancer Center, Texas 77030, USA. ${ }^{13}$ Department of Medicine, Harvard Medical School, Boston, Massachusetts 02215, USA ${ }^{14}$ Department of Pathology and Laboratory Medicine, University of North Carolina-Chape Hill, Chapel Hill, Chapel Hill, North Carolina 27599, USA. ${ }^{15}$ Department of Medicine, Vanderbilt University Medical Center, 2215 Garland Avenue, Nashville, Tennessee 37232, USA. ${ }^{16}$ Department of Pathology, University of Ulsan College of Medicine, Asan Medical Center, Seoul, 138-736, South Korea. ${ }^{17}$ Sir Peter MacCallum Cancer Department of Oncology, University of Melbourne, East Melbourne 3002, Australia. ${ }^{18}$ National Cancer Institute, Bethesda, Maryland 20892, USA. ${ }^{19}$ Division of Cancer Epidemiology and Genetics, National Cancer Institute, Bethesda, Maryland 20892, USA. ${ }^{20}$ Department of Pathology, Case Western Reserve University, Cleveland, Ohio 44106, USA. ${ }^{21}$ Department of Biomolecular Engineering and Center for Biomolecular Science and Engineering, University of California-Santa Cruz, Santa Cruz, California 95064, USA. ${ }^{22}$ Division of Gastroenterology, Department of Medicine, Duke University, Durham, North Carolina 27710, USA. ${ }^{23}$ Department of Thoracic Surgery, University of Michigan Cancer Center, Ann Arbor, Michigan 48109, USA. ${ }^{24}$ University of Pittsburgh, Pittsburgh, Pennsylvania 15213, USA. ${ }^{25}$ Department of Computer Science \& Center for Computational Molecular Biology, Brown University, 115 Waterman Street, Providence, Rhode Island 02912, USA. ${ }^{26}$ Department of Pathology, Brigham and Women's Hospital, 75 Francis Street, Boston, Massachusetts 02115, USA. ${ }^{27}$ National Cancer Center, Goyang, 410-769, Republic of 
Korea. ${ }^{28}$ The Research Institute at Nationwide Children's Hospital, Columbus, Ohio 43205, USA. ${ }^{29}$ The Genome Institute, Washington University, St Louis, Missouri 63108 USA. ${ }^{30}$ Greater Poland Cancer Centre, Garbary, 15, 61-866, Poznan, Poland. ${ }^{31} \mathrm{KU}$ Leuven, Department of Electrical Engineering-ESAT (STADIUS), Leuven, Belgium. ${ }^{32}$ Institute for Applied Cancer Science, Department of Genomic Medicine, University of Texas MD Anderson Cancer Center, Houston, Texas 77054, USA. ${ }^{33}$ The Center for Biomedical Informatics, Harvard Medical School, Boston, Massachusetts 02115, USA.

${ }^{34}$ Cancer Biology Division, Johns Hopkins University, Baltimore, Maryland 21231, USA.

${ }^{35}$ Helen Diller Family Comprehensive Cancer Center, University of California-San Francisco, San Francisco, California 94143-0128, USA. ${ }^{36}$ International Genomics Consortium, Phoenix, Arizona 85004, USA. ${ }^{37}$ Buck Institute for Research on Aging, Novato, California 94945, USA. ${ }^{38}$ Chonnam National University Medical School, Gwangju, 501-746, Republic of Korea. ${ }^{39}$ City Clinical Oncology Dispensary, Saint Petersburg 198255, Russia. ${ }^{40}$ Cureline, Inc., South San Francisco, California 94080, USA.

${ }^{41}$ Departments of Medicine and Genetics, University of North Carolina at Chapel Hill, Chapel Hill, North Carolina 27599, USA. ${ }^{42}$ Helen F. Graham Cancer Center \& Research
Institute, Christiana Care Health System, Newark, Delaware 19713, USA. ${ }^{43}$ Keimyung University School of Medicine, Daegu, 700-712, Republic of Korea. ${ }^{44}$ Ontario Tumour Bank - Hamilton site, St. Joseph's Healthcare Hamilton, Hamilton, Ontario L8N 3Z5, Canada. ${ }^{45}$ Ontario Tumour Bank - Kingston site, Kingston General Hospital,

Kingston, Ontario K7L 5H6, Canada. ${ }^{46}$ Ontario Tumour Bank, Ontario Institute for Cancer Research, Toronto, Ontario M5G OA3, Canada. ${ }^{47}$ Pusan National University Hospital, Busan, 602-739, Republic of Korea. ${ }^{48}$ Department of Pathology, Memorial

Sloan-Kettering Cancer Center, New York, New York 10065, USA. ${ }^{49}$ Department of Pathology, Duke University, Durham, North Carolina 27710, USA. ${ }^{50}$ Department of Surgery, Yonsei University College of Medicine, Seoul, 120-752, Republic of Korea. ${ }^{51}$ Department of Gastrointestinal Medical Oncology, University of Texas MD Anderson Cancer Center, Houston, Texas 77030, USA. ${ }^{52}$ SRA International, Fairfax, Virginia 22033, USA. ${ }^{53}$ Center for Biomedical Informatics and Information Technology, National Cancer Institute, Rockville, Maryland 20850, USA. ${ }^{54}$ National Human Genome Research Institute, Bethesda, Maryland 20892, USA. ${ }^{55}$ SAIC-Frederick, Inc., Frederick, Maryland 21702, USA 